\title{
A Quantum Chemical Screening of Two Imidazole-Chalcone Hybrid Ligands and Their Pd, Pt and Zn Complexes for Charge Transport and Nonlinear Optical (NLO) Properties: A DFT Study
}

\author{
Fritzgerald Kogge Bine ${ }^{1}$ Numbonui Stanley Tasheh${ }^{1}$, Julius Numbonui Ghogomu1,2* \\ ${ }^{1}$ Research Unit of Noxious Chemistry and Environmental Engineering, Department of Chemistry, Faculty of Science, University \\ of Dschang, Dschang, Cameroon \\ ${ }^{2}$ Department of Chemistry, Faculty of Science, The University of Bamenda, Bambili-Bamenda, Cameroon \\ Email: *ghogsjuju@hotmail.com
}

How to cite this paper: Bine, F.K., Tasheh, N.S. and Ghogomu, J.N. (2021) A Quantum Chemical Screening of Two Imidazole-Chalcone Hybrid Ligands and Their Pd, Pt and Zn Complexes for Charge Transport and Nonlinear Optical (NLO) Properties: A DFT Study. Computational Chemistry, 9, 215-237. https://doi.org/10.4236/cc.2021.94012

Received: July 21, 2021

Accepted: October 15, 2021

Published: October 18, 2021

Copyright $\odot 2021$ by author(s) and Scientific Research Publishing Inc. This work is licensed under the Creative Commons Attribution International License (CC BY 4.0).

http://creativecommons.org/licenses/by/4.0/

\begin{abstract}
A quantum chemical screening of two imidazole-based chalcone ligands: 2[1-(3-(1H-imidazol-1-yl)propylimino)-3-(phenylallyl)]phenol and 2-[1-(3- $(1 \mathrm{H}-$ imidazol-1-yl)propylimino)-3-4-nitrophenylallyl]phenol (hereinafter referred to as HL1 and HL2 respectively) and their Pd, Pt and Zn chelates for charge transport and nonlinear optical (NLO) properties, is reported via dispersioncorrected density functional theory (DFT-D3) and time-dependent DFT (TDDFT) methods. From our results, Pd and Pt complexes have been observed to show excellent hole-transport properties, owing to their very small reorganization energies. The light extraction efficiency of the HL1-Pt complex was deduced to be particularly impressive, thus suitable for the manufacture of hole transport layer in violet light emitting diodes (LEDs). Moreover, redox potentials and chemical stability studies have enabled us to validate the greater stability in moisture (towards oxidation), of HL2 complexes compared to their HL1 counterparts. The first and second hyperpolarizabilities of both ligands and their complexes have been found to be outstandingly higher than those of the push-pull prototypical, para-nitroaniline by factors of up to 12 in the case of HL2. These compounds, with the exception of the HL2-Pt complex, are thus interesting candidates having wide transparency tradeoffs for NLO efficiency in the manufacture of optoelectronic and photonic devices capable of second and third-order NLO response. Finally, metal chelation has been established to enhance the NLO response of all the chalcone-based imidazole ligands investigated as a result of metal-ligand charge transfer and ligand-
\end{abstract}


metal charge transfer electronic transitions identified in the resulting complexes with the exception of the zinc complexes.

\section{Keywords}

Density Functional Theory, Time-Dependent Density Functional Theory, Chalcone-Imidazoles, Charge Transport and Nonlinear Optical Properties

\section{Introduction}

Chalcone derivatives have extensively been used in optoelectronics as photorefractive polymers, nonlinear optical (NLO) materials, chromophore sensors, fluorescent probes for the determination of metal ions and sensing of DNA, and in the study of photo-alignment layers of liquid crystal displays [1] [2] [3]. As a matter of fact, these derivatives are one of the most interesting groups of materials for NLO applications because, apart from exhibiting high NLO coefficients, they are well-known for their good crystallizability into non-centrosymmetric structures with excellent second harmonic generation (SHG) conversion efficiency [1] [4] [5]. Additionally, they possess a good optical limiting behavior when in contact with nanosecond laser pulses at Nd-YAG laser wavelengths $(1064 \mathrm{~nm})$ [6]. It must also be noted that in recent times, organic materials exhibiting NLO properties have intensely been investigated in comparison with traditional inorganic nonlinear crystals because of their many superior properties such as: ultrafast response times, lower dielectric constants, high internal quantum efficiency, high damage threshold, flexible design and low driving voltage which contributes to an efficient energy usage, cognizance of the growing demand for energy, hence impacting immensely the energy budgets of countries [5] [7] [8]. Given that chalcones bear a very good synthon, a variety of novel heterocycles with good pharmaceutical and even NLO profiles dyes have been designed [9]. Chalcones incorporate a donor- $\pi$-acceptor- $\pi$-donor $(D-\pi-A-\pi-D)$ type conjugated system with appreciable hyperpolarizability, prerequisite for potential interest in NLO applications [10]. Moreover, the presence of intramolecular charge-transfer features in the chalcone skeleton, allows for the design of new molecules substituted for donor or acceptor groups, which gives us an understanding of the structure-property relationships [6]. Nevertheless, the efficiency of organic light emitting diodes (OLEDs) is relatively low, thus slowing its very ambitious economic impact, partly because of their low stability in air and moisture, low luminous efficiency and carrier mobility amongst other bottlenecks [11] [12]. For these reasons, there is a pressing need to find novel materials with efficient applicability in the field of optoelectronics and photonics. Although the biological uses of chalcones have been investigated widely in recent times, the use of chalcones as potential optoelectronic materials is still a topic of great research interest [4]. Often, in a bid to get around the reported poor luminescence properties of chalcone-based organic molecules, partly due to the quenching pro- 
cesses from the intramolecular torsional motions and cis-trans isomerization in the $\alpha, \beta$-unsaturated ketone moiety, the complexation method is often a way out towards improving their optical properties [3] [13]. Fortunately, organometallic and coordination complexes have found a place of choice for the production of NLO materials due to their tunable metal centers and their inherent chargetranfer transitions [14]. Motivated by the role played by complexation in improving the optical properties of chalcone-based compounds, we herein turn our interest on the investigation of chalcone-imidazole complexes for optoelectronic applications.

Based on fluorescence properties of some chalcone based Schiff bases of $\mathrm{N}$ (3-aminopropyl)imidazole complexes earlier investigated by Kalanithi and coworkers [3], it is envisaged that these compounds should show promising charge transport and NLO characteristics besides the fact that they are also nitrogencontaining $\pi$-conjugated derivatives [15]. Likewise, given the fact that chalconeimidazole based hybrid chromophores are less investigated in this perspective, studies in this light are very much imperative. To the best of our knowledge, studies on the impact of transition metal complexation on the OLED properties of chalcone-imidazole hybrids are scarce. In line with the fact that transition metal coordination is most likely to improve upon the mechanical properties and thermal stability of these molecules, investigations on chalcone-based complexes in this regard are appealing.

This study therefore is aimed at investigating the combined effects of the nitro group substituent and the complexation of two imidazole-based chalcone ligands: 2-[1-(3-(1H-imidazol-1-yl)propylimino)-3-(phenylallyl)]phenol (HL1) and 2-[1(3-(1H-imidazol-1-yl)propylimino)-3-4-nitrophenylallyl]phenol (HL2) (see Figure 1) on their charge transport and NLO properties. To achieve our objective, the polarizability $(\alpha)$, first hyperpolarizability $(\beta)$ and second hyperpolarizabilies $(\gamma)$ have been calculated, given that these quantities are key indicators and reflect the extent to which optical nonlinearity can be observed [16]. It must also be recalled that second-order response governed by the second order hyperpolarizability offers more varied and richer behavior than first-order NLO process due to the higher dimensionality of the frequency space [16]. Therefore, the investigation of the second-order hyperpolarizability of the chalcone derivatives investigated herein has been probed into in order to conclude on the appropriateness of the materials for NLO applications [16] [17]. Similarly, properties with respect to OLED applications such as oxidation and reduction potentials, hole $\left(\lambda_{h}\right)$ and electron reorganization $\left(\lambda_{e}\right)$ energies, electronic absorption and emission spectra, electric dipole moments have also been probed into herein.

Given the irrefutable role theoretical investigations play in the design and synthesis of materials, the density functional theory (DFT) electronic structure method and its time-dependent extension have been used as the platform for all our calculations considering the fact that the DFT method is relatively faster than other methods like the post Hatree-Fock and coupled cluster methods at comparable accuracy [18]. 

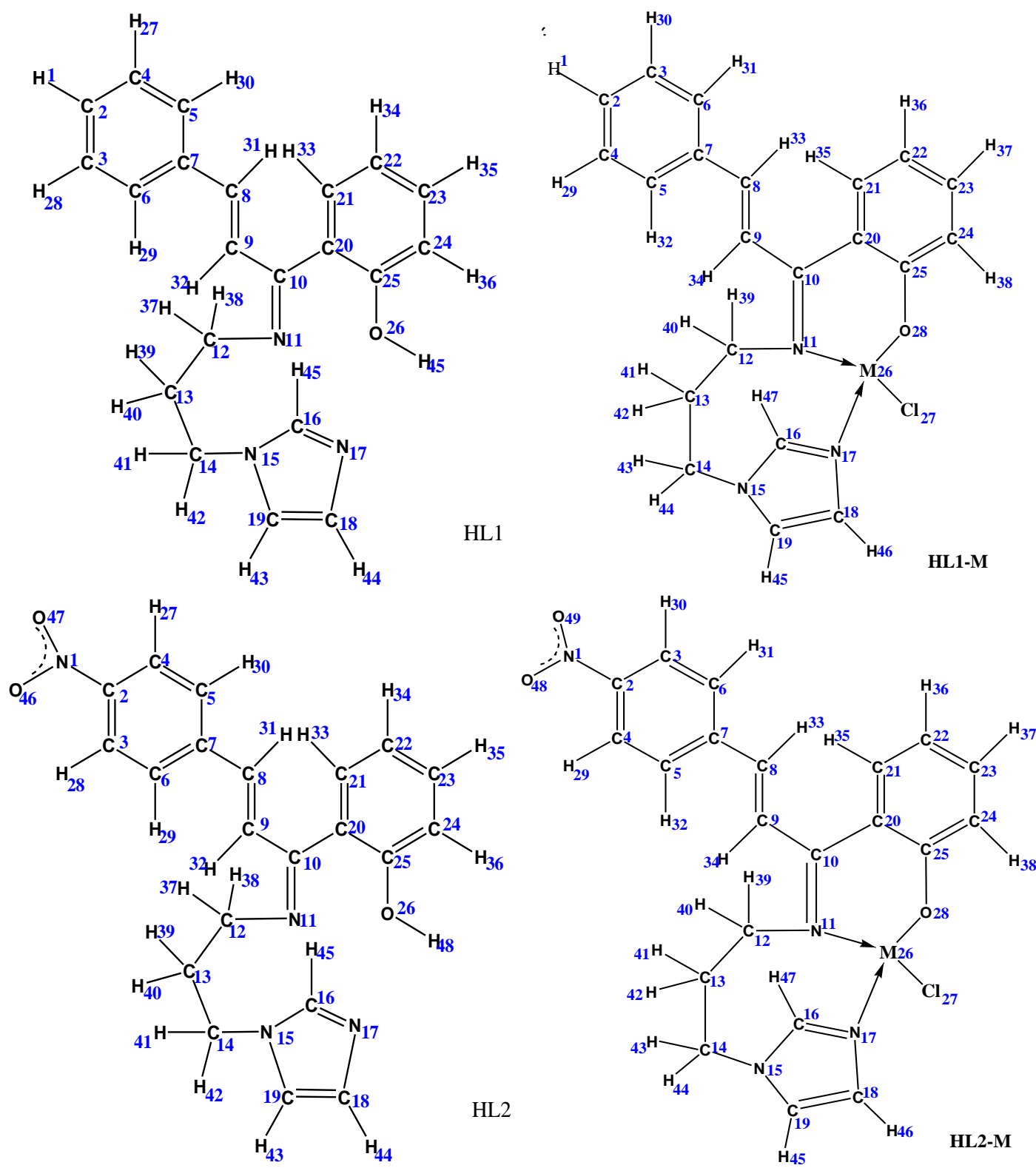

Figure 1. Input structures of studied ligands (HL1 and HL2) and complexes with $\mathrm{M}=\mathrm{Pd}^{2+}, \mathrm{Pt}^{2+} \& \mathrm{Zn}^{2+}$ and $\mathrm{Hg}^{2+}$.

\section{Computational Details}

All quantum chemical calculations in the present study were carried out using the ORCA 4 package [19], with the exception of the study of the NLO response calculations which were done with the Firefly quantum chemical package [20], which is based on part of the GAMESS US source code [21]. The GGA functional BP86 [22] [23] in conjunction with the def2-TZVP basis set [24] and the B97-3c composite electronic structure approach [24] were used for geometry optimizations and frequency calculations since they yield excellent geometries and vibrational frequencies [25] [26]. To speed-up the geometry optimization and frequency calculations, but with marginal loss in accuracy, the resolution-of- 
the-identity (RI-J) approximation [27] was used in conjunction with appropriate auxiliary basis sets. In the calculation of both fluorescence and electronic absorption spectra the TD-DFT method was invoked at the cam-B3LYP/def2-TZVP level based on the BP86/ def2-TZVP optimized structures. It should be recalled that the range-separated hybrid density functional cam-B3LYP functional [28] has been found to perform well in excited state calculations and highly recommended for molecules wherein charge transfer phenomena are significant [29]. To speed up excited states calculations with marginal loss in accuracy, the Tamm-Dancoff approximation scheme was employed. The PBE0 functional [30] was employed for single-point (SP) calculations of the molecules studied along with the basis set ma-def2-TZVP for the metal ions and ma-def2-SVP for every other element. To speed-up the calculations with the hybrid functionals, the RI-J approximation was used in combination with the chain-of-spheres (COSX) approximation, giving rise to the RIJCOSX approximation [26]. Throughout the ORCA calculations, numerical quadrature grids of at least 5 were used.

The TD-DFT method in the Firefly package was used to calculate the staticl hyper polarizabilities of both organic molecules and transition metal complexes at the PBE0/SBKJC level of theory [31] based on BP86 optimized structures. The SBKJC (Stevens/Basch/Krauss/Jasien/Cundari) valence basis set and the corresponding effective core potentials (ECPs) were used here because they are appropriate for the computation of the (hyper) polarizabilities of the metal complexes investigated, since they contain heavy transition metal ions [31]. Also, longrange dispersion interactions were incorporated via the Grimme's atom-pairwise dispersion correction using the Becke-Johnson damping scheme (D3BJ) [32] [33] as implemented in both packages.

\section{Results and Discussion}

\subsection{Thermodynamic Parameters of Complexation Reactions}

The complexes investigated herein have been modeled based on earlier studies by Kalanithi and coworkers [3] following the synthetic process depicted by Equation (1).

$$
\mathrm{HLx}+\mathrm{MCl}_{2} \rightarrow[\mathrm{M}(\mathrm{Lx}) \mathrm{Cl}]+\mathrm{HCl}
$$

where $\mathrm{x}=1$ and 2 .

To verify the coordinating affinity of the HL1 and HL2 ligands toward Hg, Pd, $\mathrm{Pt}$, and $\mathrm{Zn}$ ions, the enthalpies $\left(\Delta H_{\mathrm{Rxn}}\right)$, and Gibbs free energies $\left(\Delta G_{\mathrm{Rxn}}\right)$ at room temperature have been calculated and presented in Table 1 using Equations (2) and (3).

$$
\begin{gathered}
\Delta H_{\mathrm{Rxn}}=H_{[\mathrm{M}(\mathrm{Lx}) \mathrm{Cl}]}^{0}+H_{\mathrm{HCl}}^{0}-\left(H_{\mathrm{HLx}}^{0}+H_{\mathrm{MCl}_{2}}^{0}\right) \\
\Delta G_{\mathrm{Rxn}}=G_{[\mathrm{M}(\mathrm{Lx}) \mathrm{Cl}]}^{0}+G_{\mathrm{HCl}}^{0}-\left(G_{\mathrm{HLx}}^{0}+G_{\mathrm{MCl}_{2}}^{0}\right)
\end{gathered}
$$

Here, $H^{0}$ and $G^{0}$, respectively, represent the enthalpies and Gibbs free energies of the respective species at $298.15 \mathrm{~K}$ and $1.00 \mathrm{~atm}$, obtained at the D3(BJ)- 
BP86/def2-TZVP level of theory in gas phase.

It is clear from Table 1 that the HL1 ligand has a higher affinity for the Pd, Pt, and $\mathrm{Zn}$ than the HL2. It is also obvious from the values in Table 1 that the formation of HL1-Hg and HL2-Hg is not thermodynamically feasible at room temperature, since their $\Delta G_{\mathrm{Rxn}}$ values are positive.

Based on these results, it can be concluded that the HL1 and HL2 ligands have significantly higher affinities for the $\mathrm{d}$ metal ions $\mathrm{Pd}(\mathrm{II})$, and $\mathrm{Pt}(\mathrm{II})$ and can efficiently chelate them in chemical and biological systems, compared to the $\mathrm{Zn}$ (II) ions since they have larger $\Delta G_{\mathrm{Rxn}}$ values. Additionally, the reactions of the $\mathrm{Pd}$, $\mathrm{Pt}$, and $\mathrm{Zn}$ with the ligands are also found to be exothermal contrary to the formation of mercury complexes. Consequently, the foregoing study will focus on the $\mathrm{Pd}, \mathrm{Pt}$, and $\mathrm{Zn}$ based complexes.

\subsection{Molecular Geometry and Structural Parameters}

The optimized geometric parameters around the coordination centers of the HL1 and HL2 transition metal complexes obtained from calculations by means of the DFT based methods: RI-BP86-D3(BJ)/def2-TZVP and B97-3c, are presented in Table 2.

It can be observed that both RI-BP86-D3(BJ)/def2-TZVP and B97-3c levels of theory give similar coordination patterns and bond parameters typical of experimental values [34] [35] for similar bond types. It is evident from Figure 2 that the HL1 ligand acts as a tridentate chelate toward the $\mathrm{Pt}(\mathrm{II}), \mathrm{Pd}(\mathrm{II})$ and $\mathrm{Zn}(\mathrm{II})$ ions. While the HL1 ligand utilizes N17 atom of the imidazole ring as a donor in the HL1-Pd and HL1-Pt complexes, it is observed that the C19 atom of the imidazole ring acts as one of the donors in the HL1-Zn complex resulting in an organometallic compound. It is noticed that the C19- $\mathrm{Zn}$ bond is the longest of all metal-ligand bonds $(2.616 \AA(2.637 \AA))$, reminiscent of the weak nature of organometallic bonds. On the other hand, the HL2 ligand is found to act as a bidentate ligand toward all the metal ions, in which cases there exist no apparent

Table 1. Enthalpies ( $\Delta H_{\mathrm{Rxn}}, \mathrm{kcal} / \mathrm{mol}$ ), and Gibbs free energies $\left(\Delta G_{\mathrm{Rxn}}, \mathrm{kcal} / \mathrm{mol}\right)$ of complex formation between the HL1 and HL2 ligands and some transition metal chlorides at room temperature at the D3 (BJ)-BP86/def2-TZVP level of theory in gas phase.

\begin{tabular}{ccc}
\hline Complexes & $\Delta H_{\mathrm{Rxn}}$ & $\Delta G_{\mathrm{Rxn}}$ \\
\hline HL1-Hg & 0.959 & 0.695 \\
HL1-Pd & -67.145 & -64.905 \\
HL1-Pt & -67.395 & -65.327 \\
HL1-Zn & -20.474 & -20.439 \\
HL2-Hg & 1.995 & 1.696 \\
HL2-Pd & -65.941 & -64.568 \\
HL2-Pt & -58.432 & -57.855 \\
HL2-Zn & -13.206 & -13.584
\end{tabular}


Table 2. Some selected geometric parameters in gas phase of thermodynamically stable complexes at RI-BP86-D3 (BJ)/def2-TZVP \& B97-3c levels of theory.

\begin{tabular}{|c|c|c|c|c|c|c|}
\hline \multirow{2}{*}{$\begin{array}{c}\text { Geometric parameters } \\
\text { Bond Lengths }(\AA)\end{array}$} & \multicolumn{6}{|c|}{ BP86-D3 (BJ)/def2-TZVP (B97-3c)* } \\
\hline & HL1-Pd & HL1-Pt & HL1-Zn & HL2-Pd & HL2-Pt & HL2-Zn \\
\hline $\mathrm{M}-\mathrm{N}_{11}$ & $2.087(2.092)$ & $2.066(2.073)$ & $1.986(1.995)$ & $1.962(1.967)$ & $1.941(1.939)$ & $1.997(2.003)$ \\
\hline $\mathrm{M}-\mathrm{O}_{28}$ & $1.994(1.999)$ & $1.997(2.003)$ & $1.891(1.899)$ & $1.961(1.962)$ & $1.982(1.980)$ & $1.886(1.894)$ \\
\hline $\mathrm{M}-\mathrm{Cl}_{27}$ & $2.285(2.306)$ & $2.294(2.312)$ & $2.180(2.198)$ & $2.286(2.309)$ & $2.282(2.299)$ & $2.155(2.172)$ \\
\hline $\mathrm{M}-\mathrm{C}_{19}$ & l & l & $2.616(2.637)$ & / & / & / \\
\hline $\mathrm{M}-\mathrm{N}_{17}$ & $2.072(2.089)$ & $2.032(2.043)$ & I & I & 1 & I \\
\hline \multicolumn{7}{|l|}{ Bond Angles ( ${ }^{\circ}$ ) } \\
\hline $\mathrm{O}_{28}-\mathrm{M}-\mathrm{N}_{11}$ & $88.3(87.2)$ & $90.3(89.6)$ & $98.4(98.2)$ & $92.5(91.7)$ & $92.4(92.5)$ & $97.4(97.3)$ \\
\hline $\mathrm{N}_{11}-\mathrm{M}-\mathrm{Cl}_{27}$ & $175.9(175.5)$ & $176.6(176.5)$ & $117.4(118.4)$ & $104.5(104.8)$ & $108.7(108.6)$ & $117.3(118.0)$ \\
\hline $\mathrm{Cl}_{27}-\mathrm{M}-\mathrm{N}_{17}$ & $89.6(89.5)$ & $91.1(90.7)$ & I & I & I & I \\
\hline C19-M-O28 & I & I & l & I & I & I \\
\hline \multicolumn{7}{|l|}{ Dihedral Angles $\left({ }^{\circ}\right)$} \\
\hline $\mathrm{O}_{28}-\mathrm{M}-\mathrm{Cl}_{27}-\mathrm{N}_{11}$ & $67.8(58.3)$ & $82.6(78.6)$ & $140.2(137.7)$ & $179.0(176.9)$ & $-178.9(-180.0)$ & $147.7(142.2)$ \\
\hline $\mathrm{O}_{28}-\mathrm{M}-\mathrm{Cl}_{27}-\mathrm{N} 17$ & $178.0(178.0)$ & $178.4(178.1)$ & / & I & 1 & I \\
\hline $\mathrm{Cl}_{27}-\mathrm{M}-\mathrm{N}_{17}-\mathrm{N}_{11}$ & $176.1(176.1)$ & $176.6(176.5)$ & I & I & l & I \\
\hline $\mathrm{Cl}_{27}-\mathrm{M}-\mathrm{C} 19-\mathrm{N} 11$ & I & I & $117.5(118.4)$ & l & I & I \\
\hline
\end{tabular}

* Geometric parameters in gas phase of complexes studied at B97-3c are reported in parenthesis.

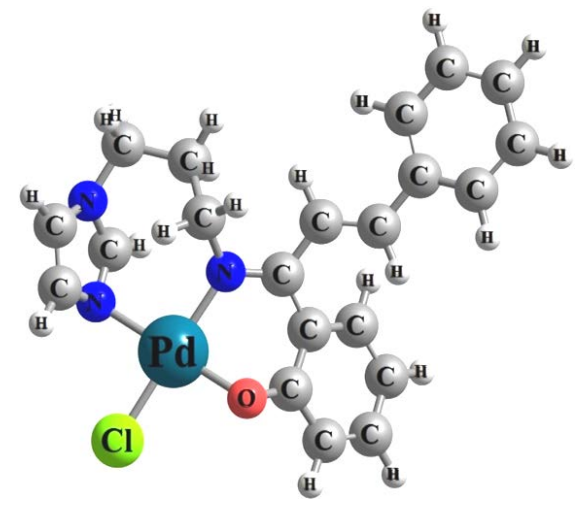

HL1-Pd

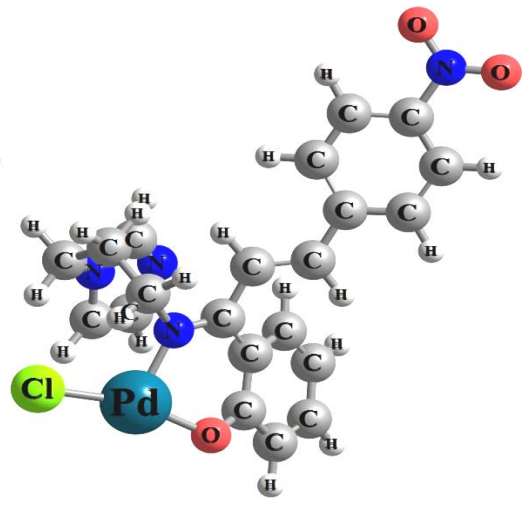

HL2-Pd

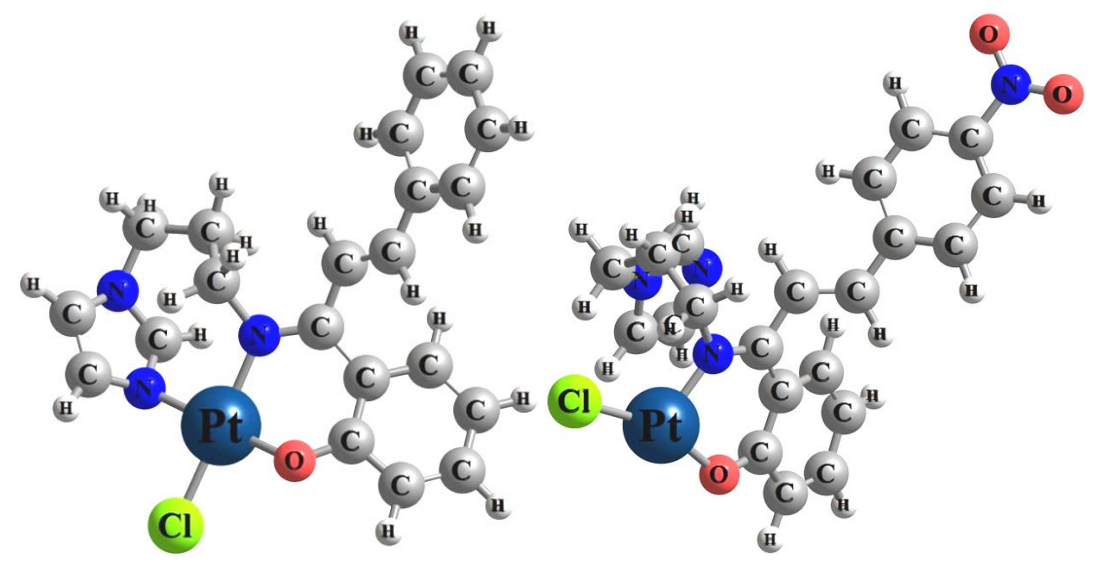

HL1-Pt 


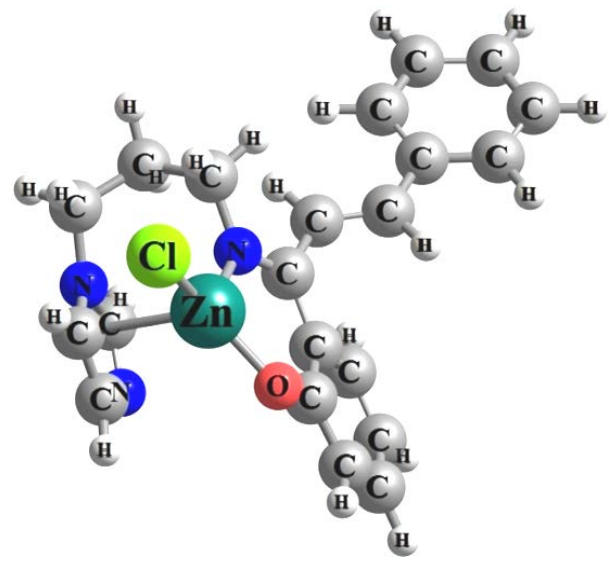

HL1-Zn

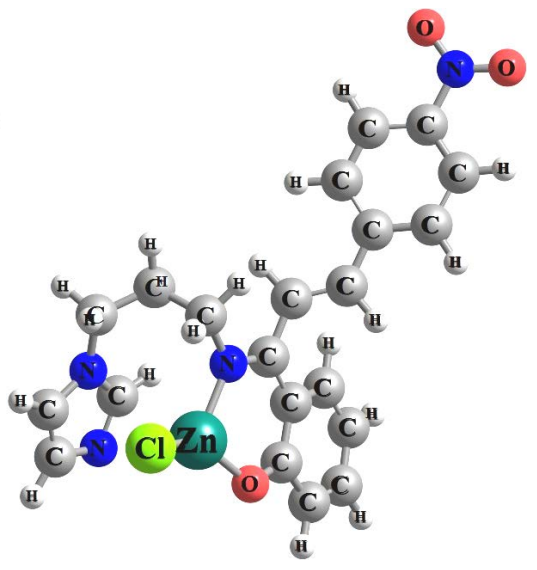

HL2-Zn

Figure 2. Optimized structures of studied HL1 and HL2 complexes in gas phase at the RI-BP86-D3 (BJ)/def2-TZVP level of theory.

imidazole-based donor atom upon complexation. From the values of the bond angles and torsional angles it is evident that the HL1-Pd and HL1-Pt complexes have distorted square planar geometries around the central metals. The HL1-Zn on its part shows a distorted tetrahedral geometry while the HL2 complexes are found to have nearly perfect trigonal planar geometries with the exception of the HL2-Zn complexes in which the torsional angle $\left(\mathrm{O}_{28}-\mathrm{M}-\mathrm{Cl}_{27}-\mathrm{N}_{11}\right)$ deviates considerably from perfect planarity.

A correlation of both methods with respect to the obtained bond parameters permits the establishment of the correlation Equation (4);

$$
y=1.000 x-1.174
$$

where $y=\mathbf{B} 97-3 \mathbf{c}$ bond parameters,

$x=$ BP86 bond parameters.

The correlation coefficient $\left(R^{2}=0.998\right)$ obtained between the bond parameters obtained at the aforementioned levels shows a good linear relationship between both methods, thus affirming somewhat perfect similarity of both levels of theory used for geometry optimization and frequency calculations. Consequently, in the present study geometries obtained at both levels of theory will be used at our convenience.

\subsection{OLED Properties}

The percolation theory or hopping theory, used to describe the electronic behavior of devices, has been in recent times an important research tool for a rational insight in the area of organic electronics [36]. In the search for appropriate OLED materials with high efficiency, charge mobility across molecular crystals, consisting of successive hopping from molecule to molecule, remains an indisputable factor, amongst other important criteria, and relies on two essential parameters, viz.: the intermolecular electronic coupling, also called electron transfer integral, $H$, and the reorganization energy of the electron transfer process between a molecular dimer, $\lambda$, which is well illustrated in the Marcus rate equa- 
tion of electron or hole-transfer, $k_{e t}$ [37] [38] as follows:

$$
k_{e t}=\left(\frac{4 \pi^{2}}{h}\right) \Delta H_{a b}^{2}\left(4 \pi \lambda K_{B} T\right)^{-\frac{1}{2}} \exp \left(\frac{-\left(\Delta G^{0}+\lambda\right)^{2}}{4 \lambda K_{B} T}\right)
$$

where $\lambda$ and $\Delta H_{a b}$ are the reorganization energy for the intramolecular electron transfer and the electronic coupling integral between donor-acceptor pairs, respectively, and $\Delta G$ is the Gibbs free energy change of the process. Mobility is the measure of the rate of charge transport in response to an electronic field. Hence, for a significant charge transport, low reorganization and electronic coupling energy is fundamental. In view of a comparative study, $N, N^{\prime}$-diphenyl- $N, N^{\prime}$-bis (3-methlphenyl)-(1,1'-biphenyl)-4,4'-diamine(TPD) $\left(\lambda_{h}=0.290 \mathrm{eV}\right)$ and tris(8hydroxyquinolinato)aluminum(III) (Alq3) $\left(\lambda_{e}=0.276 \mathrm{eV}\right)$ which are typical hole and electron transport materials [11] respectively, have been referred to herein. The reorganization energy is composed of two parts, namely: the internal reorganization $\left(\lambda_{i}\right)$ and the external polarization $\left(\lambda_{e}\right)$. The former term $\lambda_{i}$ reflects the change in molecular geometry associated with going from the neutral state to the ionized state, and vice versa while the latter term $\lambda_{e}$ describes the change in electronic polarization of the surrounding molecules [39]. The external contribution, we must recall, is difficult to evaluate theoretically and besides, the computed values of the external reorganization energy are much smaller than their internal counterparts [40]. In the present study therefore, we focus attention on the calculation of the internal reorganization energy from first principle. The reorganization energies for the hole and electron transfers have consequently been evaluated at the RIJCOSX-PBE0-D3/ma-def2-TZVP (for the metal ion) and madef2-SVP (for the other elements)// B97-3c levels of theory in gas phase using the following formulas in Equation (6) and (7) [12] [41] and reported in Table 3.

Table 3. Computed chemical hardness $(\eta)$, adiabatic electron affinity $(A E A)$, vertical electron affinity $(V E A)$, vertical ionization potential $(I P(V))$, adiabatic ionization potential $(I P(a)), \lambda_{\mathrm{e}}$ and $\lambda_{\mathrm{h}}$ values in $\mathrm{eV}$ of the HL1 and HL2 complexes at PBE0-D3/madef2-TZVP (for the metal ion) and ma-def2-SVP (for the other elements)//B97-3c levels of theory in gas phase.

\begin{tabular}{cccccccc}
\hline Compounds & $\eta$ & $I P(v)$ & $I P(a)$ & $A E A$ & $V E A$ & $\lambda_{h}$ & $\lambda_{e}$ \\
\hline HL1 & 1.186 & - & - & - & - & 3.203 & 0.800 \\
HL1-Pd & 2.762 & 6.957 & 6.821 & 1.662 & 1.433 & 0.202 & 0.439 \\
HL1-Pt & 2.682 & 6.731 & 6.635 & 1.667 & 1.366 & 0.140 & 0.546 \\
HL1-Zn & 3.068 & 7.389 & 7.075 & 1.674 & 1.253 & 0.572 & 0.856 \\
HL2 & 0.995 & - & - & - & - & 3.019 & 0.673 \\
HL2-Pd & 2.634 & 7.750 & 7.600 & 2.789 & 2.482 & 0.236 & 0.597 \\
HL2-Pt & 2.661 & 7.625 & 7.447 & 2.478 & 2.303 & 0.275 & 0.410 \\
HL2-Zn & 2.754 & 7.678 & 7.223 & 2.755 & 2.171 & 0.673 & 0.738 \\
\hline
\end{tabular}

${ }^{\star}$ Experimental electron affinity $(E A)$ of the Oxygen molecule $=0.448 \pm 0.006 \mathrm{eV}[42]$. 


$$
\begin{aligned}
& \lambda_{\mathrm{e}}=\left(E_{0}^{-}-E_{-}^{-}\right)+\left(E_{-}^{0}-E_{0}^{0}\right) \\
& \lambda_{\mathrm{h}}=\left(E_{0}^{+}-E_{+}^{+}\right)+\left(E_{+}^{0}-E_{0}^{0}\right)
\end{aligned}
$$

where $E_{0}^{+}\left(E_{0}^{-}\right)$is the single point energy of the cation (anion) calculated with the optimized structure of the neutral molecule. Similarly, $E_{+}^{+}\left(E_{-}^{-}\right)$is the single point energy of the cation (anion) calculated with the optimized cation (anion) structure, $E_{+}^{0}\left(E_{-}^{0}\right)$ is the single point energy of the neutral molecule calculated at the cationic (anionic) state. Finally, $E_{0}^{0}$ is the single point energy of the neutral molecule at the ground state.

It is observed from Table 3 that $\lambda_{h}$ (reorganization energy for hole transport) values are comparatively smaller as compared to the $\lambda_{e}$ (reorganization energy for electron-transport) values demonstrating relevance of studied complexes as p-type materials for organic light emitting devices. Moreover, the $\lambda_{e}$ values obtained are found to be greater than that of Alq3. Amongst the studied complexes, the $\mathrm{Pt}(\mathrm{II})$ and $\mathrm{Pd}(\mathrm{II})$ complexes have been found to present interestingly lower $\lambda_{h}$ values compared to TPD, suggestive of their suitability as p-type materials for OLEDs. The trend in $\lambda_{h}$ values is found to be; HL2-Zn $>$ HL1-Zn $>$ HL2-Pt $>$ HL2-Pd > HL1-Pd > HL1-Pt, implying that among the molecules studied, HL1-Pt complex possesses the best hole transport properties since its $\lambda_{h}$ value $(0.140 \mathrm{eV})$ is significantly smaller that of the prototypical molecule, TPD. It is worthy of note that hole-transport capability in the present study is significantly improved upon metalleation of the HL1 and HL2 ligands by d transition metals. It should be recalled that low reorganization energies are desirable in charge transport materials as they result in greater charge mobility due to smaller hopping barriers.

\subsubsection{Redox Potentials $\backslash$ Chemical Stability of Studied Compounds}

One of the key characteristics of an ideal optical crystal used in fields like photonics and optoelectronics is its chemical stability. In our work therefore, in view of predicting the stability of our chemical systems, the absolute hardness $(\eta)$, have been calculated based on the molecular electron density theory (MEDT) [41], using Equation 8:

$$
\left(\frac{\partial^{2} E}{\partial N^{2}}\right)_{v(r)}=\eta=\frac{1}{2}\left(I P_{v}-E A_{v}\right)
$$

In Equation (8), $v(r)$ represents the external potential of an $\mathrm{N}$-electron system with total energy $E$. IP and $E A$ are the first vertical ionization potential and electronic affinity of the system, respectively. The values of $I P$ and $E A$ are calculated as follows:

$$
\begin{gathered}
I P_{v}=E_{N-1}-E_{N} \\
E A_{v}=E_{N}-E_{N+1}
\end{gathered}
$$

where $E_{N}, E_{N-1}$ and $E_{N+1}$ are the single point energies of the neutral, cationic, and anionic forms of the $N$-electron system for the optimized geometry of the neutral molecule. 
It is clear from Table 3 that the complexes are significantly more stable chemically than the ligands. Also, it is observed that as far as the complexes are concerned, the $\mathrm{Zn}(\mathrm{II})$ complexes are more stable than their $\mathrm{Pt}(\mathrm{II})$ and $\mathrm{Pd}(\mathrm{II})$ counterparts.

Another important factor which also characterizes the charge transport properties of OLED materials is their ionization potential and electron affinity. In an electron transport material, the electron affinity must be high enough to permit an efficient injection of electrons into low-lying unoccupied molecular orbitals [43]. In this work, the ionization potentials $(I P)$ and electron affinities $(E A)$ have been calculated according to Equations (8)-(11) at PBE0-D3(BJ)/ma-def2-TZVP (for the metal ion) and ma-def2-SVP (for the other elements) level of theory in gas phase.

$$
\begin{aligned}
& I P(v)=E_{0}^{+}-E_{0}^{0} \\
& I P(a)=E_{+}^{+}-E_{0}^{0} \\
& A E A=E_{-}^{-}-E_{0}^{0} \\
& V E A=E_{0}^{-}-E_{0}^{0}
\end{aligned}
$$

The calculated $E A$ values for the studied complexes are all greater than the calculated $E A$ of oxygen (see Table 3 ) defining their anionic stability toward the oxygen present in surrounding. Thus, according to these observations the HL2 complexes are quite stable to air moisture compared to their HL1 counterparts. Also, the ionization energy (IE) of the hole transport layer (HTL) is one important material property that will influence the open-circuit voltage, fill factor, and short-circuit current [44]. On the basis of above theoretical calculations, the ionization potential values of the complexes follow the trend HL1-Pt $<$ HL1-Pd $<$ HL1-Zn < HL2-Pt $<$ HL2-Zn $<$ HL2-Pd. The HL2-Pd and HL2-Zn complexes score the highest $I P$ values while the HL1-Pt and HL1-Pd have shown the least values. Upon application of these complexes in optoelectronics, the present study on IP values will be very informative on the ideal HTL IP range within which holes can be injected into the emissive layer of these potential hole transport candidates.

\subsubsection{Electronic Absorption $\backslash$ Emission Spectra and Charge Transport}

It is worth recalling that a good charge transport material should not absorb in regions of the spectrum where the pixel is emitting light. This is important because photons of light can go through a number of internal reflections before exiting the device. Thus, even a small amount of absorption in the wrong region of the spectrum can lead to a drastic decrease in the light out coupling efficiency of the OLED device (Light extraction efficiency) [8]. Therefore, a comprehensive study of absorption and emission spectra of these materials allows for their rational design in a bid to achieve marginal absorption in their emission window (achieve high stoke shifts). In this study therefore, electronic absorption and fluorescence studies of studied compounds have been undertaken for a rational design of efficient optoelectronic devices. 
Herein, the HL2-Pt, HL2-Pd, HL1-Pd and HL1-Pt complexes earlier observed to be potential hole transport materials, have been further screened with respect to their electronic absorption and fluorescence spectra calculated at the camB3LYP/def2-TZVP//D3(BJ)-BP86/def2-TZVP levels of theory. From Figure 3, it can be observed that in the HL1-Pt complex, there is very little absorption $\left(\lambda_{\max }=333 \mathrm{~nm}\right)$ in the emission window $\left(\lambda_{\max }=411.64 \mathrm{~nm}\right)$ of the complex, conferring on it the status of a good hole transport material and can potentially serve in the design of hole transport layers (HTL) in the fabrication of violet OLEDs. The HL1-Pd compound on the other hand is observed to emit a low light intensity though somewhat out of the absorption window. In the HL2-Pd complex, though the absorption window does not coincide neatly with the fluorescence spectrum, the latter remains relatively very weak and will require considerable redesigning to boost its fluorescence properties. In the case of the HL2-Pt complex, it is observed that the emission window falls sharply within its absorption window. Consequently, a severe decrease in the light out coupling efficiency of the device is expected even though it possesses a good hole reorganization energy. Consequently, in the redesign of HL2-Pt as a hole transport material, emphasis must be directed towards improving on its light absorption and emission characteristics.

\subsection{NLO Response}

Quantum chemical calculations have been shown to be useful within the description of the relationship among the electronic structure of chemical systems and their NLO response [43] [45]. In this study, the combined effects of nitration and transition metal complexation of the HL1 parent molecular framework on the NLO response have been investigated. The calculation of the components of the static electronic linear polarizability $(\alpha)$, the first hyperpolarizability $(\beta)$ and the second hyperpolarizability $(\gamma)$ tensors at the PBE0/SBKJC//BP86/def2-TZVP level of theory, were done via the TD-DFT method as implemented in the Firefly quantum chemical package [19].

In general, nonlinear optical phenomena occur whenever the optical fields associated with one or more laser beams propagating in a material are large enough to produce polarization fields proportional to the product of two or more of the incident fields [46]. However, linking the macroscopic coefficients to the individual molecular coefficients remains a significant problem because of interactions between neighboring molecules. However, most often the macroscopic second-order nonlinearities of organic materials can rationally be described by the nonlinearities of the constituent molecules [47]. Hence, polarization $P$, induced in an atom or a molecule by an external field $E$ can be given by Equation 15, where the vector quantities $P$ and $E$ are related by the tensor quantities $\alpha, \beta$, and $\gamma$.

$$
P=\alpha E+\beta E^{2}+\gamma E^{3}+\cdots+q^{(1)} \Delta E+q^{(2)} \Delta E^{2}+\cdots
$$

where $q^{(n)}$ is the nth quadrupole nonlinear susceptibility. 

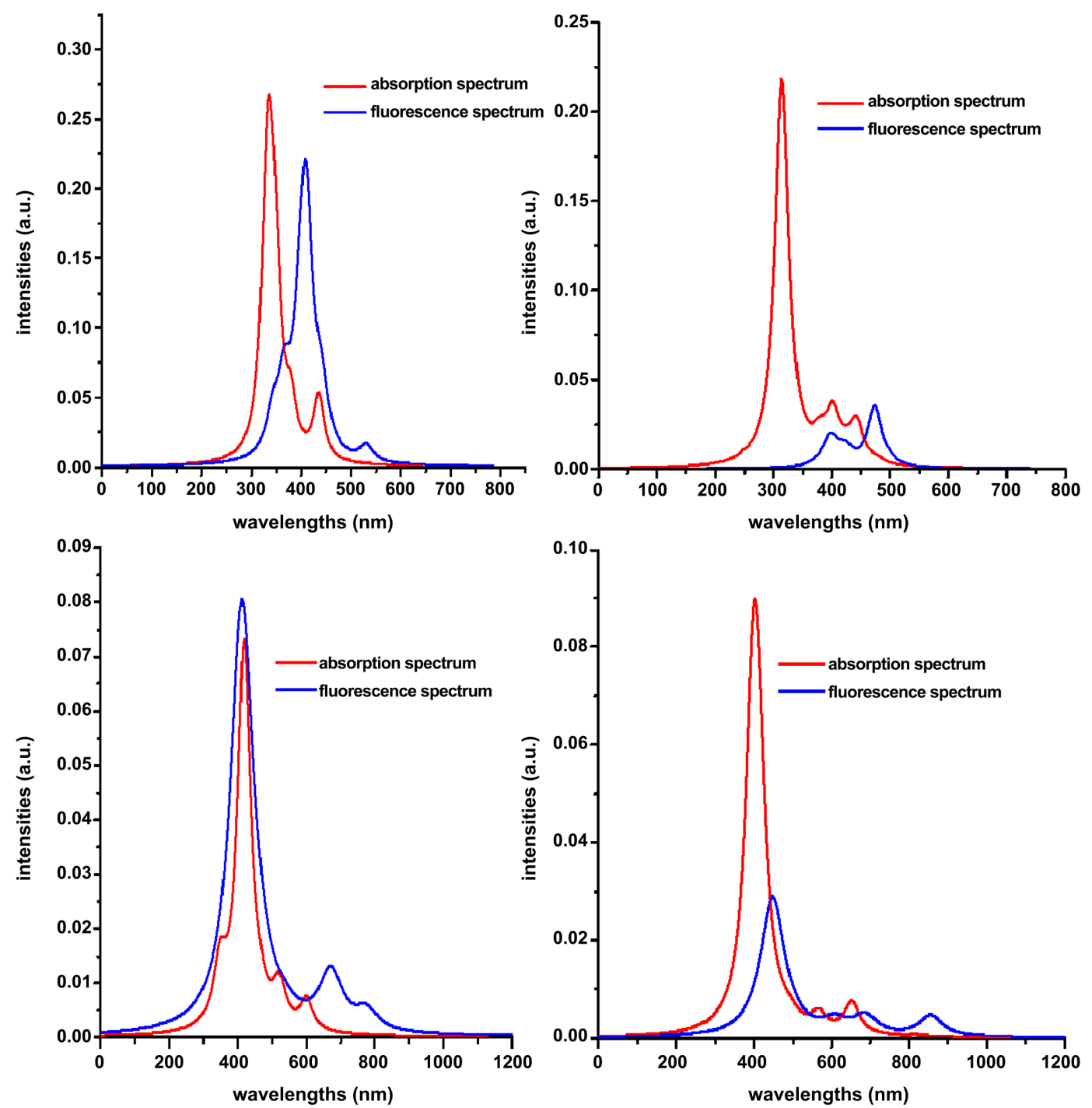

Figure 3. The electronic absorption and fluorescence spectra of HL1-Pd, HL1-Pt, HL2-Pd and HL2-Pt in gas-phase at cam-B3LYP/def2-TZVP level of theory.

The definition for the average linear polarizability $\langle\alpha\rangle$, the total static first hyperpolarizability ( $\beta_{\text {tot }}$ ), the vector part of the dynamic first hyperpolarizability $\left(\beta_{-v}\right)$, and the averaged (isotropic) third hyperpolarizability $\langle\gamma\rangle$ were calculated using the following equations:

$$
\begin{aligned}
& \langle\alpha\rangle=\frac{1}{3}\left(\alpha_{x x}+\alpha_{y y}+\alpha_{z z}\right) \\
& \beta_{\text {tot }}=\left(\beta_{x}^{2}+\beta_{y}^{2}+\beta_{z}^{2}\right)^{1 / 2}
\end{aligned}
$$

where: $\beta_{x}=\left(\beta_{x x x}+\beta_{x y y}+\beta_{x z z}\right) ; \beta_{y}=\left(\beta_{y y y}+\beta_{y z z}+\beta_{y x x}\right)$;

$$
\beta_{z}=\left(\beta_{z z z}+\beta_{z x x}+\beta_{z y y}\right)
$$

$$
\beta_{-v}=\left(\beta_{x}^{2}+\beta_{y}^{2}+\beta_{z}^{2}\right)^{1 / 2}
$$

where $\beta_{i}(i=x, y, z)$ is given by: 


$$
\begin{gathered}
\beta_{i}=\left(\frac{1}{3}\right) \sum_{j=x, y, z}\left(\beta_{i j j}+\beta_{j i j}+\beta_{j j i}\right) \\
\langle\gamma\rangle=\frac{1}{5}\left[\gamma_{x x x x}+\gamma_{y y y y}+\gamma_{z z z z}+2\left(\gamma_{x x y y}+\gamma_{x x z z}+\gamma_{y y z z}\right)\right]
\end{gathered}
$$

From Tables 4-7, an enhancement in the NLO properties have been observed after the introduction of nitro-group (withdrawing group) into HL1 ligand framework to obtain HL2. This change may be explained from the fact that the nitro group increases the polarizability of the molecule as observed in Table 5, favoring charge transfer. Hence, the static first hyperpolarizabilities of the HL2 ligand are found to be 7.5 and 2.5 times higher than HL1 and paranitroaniline (a prototypical molecule) respectively. Moreover, the static second hyperpolarizabilities of the HL2 ligand are found to be 2.6 and 12 times higher than HL1 and paranitroaniline, which is a motivating prerequisites for the use of HL2 as a NLO material.

Table 4. Static average polarizabilities ( $\alpha$ in $10^{-25}$ esu), along with some tensor components (in a.u.) for ligands, their transition metal complexes and $p$-NA, calculated at $\omega=0$ using the TD-DFT method at PBE0/SBKJC level of theory.

\begin{tabular}{cccccccc}
\hline Molecules & $\alpha_{x x}$ & $\alpha_{x y}$ & $\alpha_{y y}$ & $\alpha_{z x}$ & $\alpha_{y z}$ & $\alpha_{z z}$ & $\langle\alpha\rangle \times 10^{-25} \mathrm{esu}$ \\
\hline HL1 & 288.03 & -26.73 & 280.67 & -16.33 & -23.12 & 178.93 & 369.31 \\
HL1-Pd & 387.64 & -37.61 & 374.41 & 46.23 & -53.92 & 186.99 & 468.79 \\
HL1-Pt & 392.53 & -38.20 & 383.92 & 45.54 & -52.44 & 190.32 & 477.55 \\
HL1-Zn & 339.04 & -25.97 & 302.97 & 21.75 & -64.63 & 206.91 & 419.34 \\
HL2 & 329.41 & -51.31 & 315.18 & -12.18 & -32.00 & 186.17 & 410.37 \\
HL2-Pd & 390.22 & -40.41 & 361.66 & 41.19 & -89.46 & 235.19 & 487.58 \\
HL2-Pt & 393.08 & -44.30 & 356.20 & 42.02 & -89.61 & 245.27 & 491.28 \\
HL2-Zn & 386.96 & -53.98 & 355.85 & 20.18 & -56.68 & 191.96 & 461.74 \\
p-NA & 141.93 & 0.03 & 92.66 & 0.54 & -0.33 & 29.92 & 130.66 \\
\hline
\end{tabular}

Table 5. Static total first hyperpolarizabilities ( $\beta_{\text {tot }}$ in $10^{-30} \mathrm{esu}$ ), alongside some of its components (in a.u.) for ligands, their transition metal complexes and $p$-NA, calculated at $\omega=0$ using the TD-DFT method at PBE0/SBKJC level of theory.

\begin{tabular}{cccccccc}
\hline Molecules & $\beta_{x x x}$ & $\beta_{y y y}$ & $\beta_{z z}$ & $\beta_{x}$ & $\beta_{y}$ & $\beta_{z}$ & $\beta_{\text {tot }} \times 10^{-30} \mathrm{esu}$ \\
\hline HL1 & -105.78 & 59.30 & 11.57 & -351.70 & 419.48 & -136.75 & 4.87 \\
HL1-Pd & 945.18 & 266.65 & 171.00 & 1525.35 & 453.20 & 428.95 & 14.24 \\
HL1-Pt & 1448.95 & -449.41 & 165.38 & 3158.91 & -965.23 & 1021.45 & 29.87 \\
HL1-Zn & 234.35 & 103.54 & 189.17 & -206.86 & 806.01 & -188.32 & 7.37 \\
HL2 & 1610.01 & -1466.46 & 7.76 & 3090.88 & -2854.87 & 567.49 & 36.68 \\
HL2-Pd & 1936.94 & -1734.73 & -82.43 & 3262.81 & -3087.65 & 205.14 & 38.85 \\
HL2-Pt & 2130.84 & -1590.45 & -15.63 & 3494.20 & -3242.46 & 584.50 & 41.49 \\
HL2-Zn & 2701.90 & -1159.83 & 144.49 & 3610.47 & -2065.34 & 412.47 & 36.11 \\
p-NA & -1940.14 & 0.21 & -4.09 & -1756.29 & -1.31 & -67.84 & 15.18 \\
\hline
\end{tabular}


Table 6. Isotropic values of the vector component of the first hyperpolarizbility $\left(\beta_{-v}\right.$ in $10^{-30} \mathrm{esu}$ ), alongside some of its components (in a.u.) for $\mathrm{L}$, its transition metal complexes and $p$-NA, calculated at $\omega=0$ using the TD-DFT method at PBE0/SBKJC level of theory.

\begin{tabular}{cccccc}
\hline Molecules & $\beta_{x}$ & $\beta_{y}$ & $\beta_{z}$ & $\beta_{-v} \times 10^{-30}$ esu & Dipole moment \\
\hline HL1 & -1055.10 & 1258.43 & -410.26 & 14.62 & 4.88 \\
HL1-Pd & 4576.04 & 1359.61 & 1286.86 & 42.71 & 14.71 \\
HL1-Pt & 9476.73 & -2895.70 & 3064.34 & 89.61 & 14.50 \\
HL1-Zn & -620.59 & 2418.04 & -564.97 & 22.11 & 8.82 \\
HL2 & 9272.63 & -8564.63 & 1702.48 & 110.04 & 4.40 \\
HL2-Pd & 9788.44 & -9262.95 & 615.42 & 116.55 & 0.91 \\
HL2-Pt & $10,482.61$ & -9727.37 & 1753.51 & 124.47 & 0.71 \\
HL2-Zn & $10,831.40$ & -6196.02 & 1237.40 & 108.33 & 4.16 \\
p-NA & -5268.88 & -3.92 & -203.51 & 45.55 & 7.80 \\
\hline
\end{tabular}

Table 7. Average static second hyperpolarizabilities ( $\gamma$ in $10^{-37} \mathrm{esu}$ ) and its components (in a.u.) for the ligands, their transition metal complexes and $p$-NA, calculated at $\omega=0$ using the TD-DFT method at PBE0/SBKJC level.

\begin{tabular}{cccccccc}
\hline Molecules & $\gamma_{x x x x}$ & $\gamma_{y y y y}$ & $\gamma_{z z z z}$ & $\gamma_{x x y y}$ & $\gamma_{x х z z}$ & $\gamma_{y y z z}$ & $\langle\gamma\rangle \times 10^{-37}$ esu \\
\hline HL1 & $99,211.37$ & $120,021.64$ & 9676.43 & $80,491.59$ & 9011.81 & $11,109.88$ & $86,027.20$ \\
HL1-Pd & $466,156.43$ & $235,917.57$ & $14,445.13$ & $208,845.62$ & $21,938.72$ & $23,530.87$ & $245,029.91$ \\
HL1-Pt & $552,939.80$ & $317,418.49$ & $14,229.04$ & $331,865.81$ & $32,865.25$ & $27,613.64$ & $333,855.34$ \\
HL1-Zn & $216,879.65$ & $88,478.69$ & $23,381.57$ & $87,656.10$ & $16,929.00$ & $14,962.16$ & $113,566.89$ \\
HL2 & $279,154.07$ & $285,536.77$ & 8933.56 & $253,145.65$ & $17,236.27$ & $16,973.91$ & $229,667.21$ \\
HL2-Pd & $621,111.59$ & $323,313.86$ & $42,336.54$ & $330,717.51$ & $68,391.50$ & $59,016.64$ & $380,602.66$ \\
HL2-Pt & $660,309.99$ & $308,089.10$ & $45,887.12$ & $339,861.28$ & $80,570.35$ & $66,638.33$ & $397,685.23$ \\
HL2-Zn & $684,671.50$ & $323,592.82$ & $15,283.80$ & $238,044.06$ & $19,297.26$ & $18,575.93$ & $315,076.52$ \\
p-NA & $93,093.09$ & 5077.05 & 552.39 & -2989.74 & -38.22 & 324.20 & $18,663.00$ \\
\hline
\end{tabular}

Upon metalleation of both ligands, an overall enhancement of $\gamma$ values is observed. This trend is equally noticed for the $\beta$ values with the exception of the HL2-Zn complex that witnesses a slight drop in the $\beta$ value. Also, it is observed that the HL1-Pd and HL1-Zn complexes show $\beta$ values that are inferior to that of paranitroaniline. In addition to HL1-Pt therefore, the HL2 ligand and all the HL2 complexes have been found to show high prospects to be used as NLO materials given that their $\beta$ and $\gamma$ values are greater than that of the prototype paranitroaniline and comparative to $\beta_{\text {tot }}$ values of $[\mathrm{Ru}(\mathrm{L}) 3] 2+$ complexes earlier reported [14].

The dominant components of $\beta$ in the HL2 compounds are $\beta_{x}$ and $\beta_{y}$ implying that the intramolecular charge transfer phenomenon in the compounds occurs mainly in the $\mathrm{x}$-y-direction as opposed to the HL1 compounds wherein the $\beta_{x}$ is dominant in the HL1-Pd and HL1-Pt complexes while the $\beta_{y}$ component is dominant in the HL1 and its $\mathrm{Zn}(\mathrm{II})$ complex. On the other hand, the tensor 
components of $\gamma$ for the compounds are found to contribute positively and in all directions to the average value, showing that the compounds can exhibit multi-dimensional third order NLO responses.

In view of the fact that dipole moment plays an important role in molecular polarizabilities [48], a careful examination of Table 6 on the relationship between the observed $\beta_{-v}$ values and the corresponding dipole moments has been carried out. It can be observed that the HL1 compounds show a more or less direct relationship between these values, whereas the HL2 compounds show an inverse relationship between their dipole moments and observed $\beta_{-v}$ upon transition metal complexation. Therefore, in the process of modeling derivatives (complexes) from HL1 or HL2 ligands aimed at boosting their $\beta_{-v}$ values, an increase in the dipole moment of the HL1 derivative or a reduction of dipole moment of the HL2 derivatives will be a good preliminary towards the search of much more performant NLO materials.

\section{Electronic Absorption Spectra of HL1, HL2 and Their Transition Metal Complexes}

Analysis of electronic absorption spectra results allows us to assign the character of the main transition involved in a molecule upon an external excitation process and also to gain further insight into the intramolecular charge transfer (ICT) phenomena occurring in these structures. Calculated wavelength, oscillator strength $(f)$ and molecular orbital excitations for the transition of the UV-Vis absorption bands of studied compounds are reported in Table 8. Herein, natural transition orbitals (NTO) have been computed to facilitate our discussion, a scheme that often provides a more convenient representation of the electronic transitions.

Table 8. Absorption energies $(\Delta E, \mathrm{eV})$, wavelengths $(\lambda, \mathrm{nm})$, and oscillator strengths $(f)$, contributions of the natural transition orbitals (NTOs) calculated at Cam-B3LYP/def2-TZVP level of theory in gas-phase by the TD-DFT method.

\begin{tabular}{|c|c|c|c|c|c|}
\hline Molecule & state & $\lambda_{\max }$ & $f$ & $\Delta E(\mathrm{eV})$ & electronic transition \\
\hline HL1 & 5 & 251.4 & 0.024 & 4.933 & $\begin{array}{l}\mathrm{P} \rightarrow \mathrm{H}(\mathrm{HOMO} \rightarrow \mathrm{LUMO}) 61 \% \\
(\mathrm{HOMO}-1 \rightarrow \mathrm{LUMO}+1) 38 \%\end{array}$ \\
\hline HL1-Pd & 6 & 314.2 & 0.217 & 3.946 & $\begin{array}{l}\mathrm{P} \rightarrow \mathrm{H}(\mathrm{HOMO} \rightarrow \mathrm{LUMO}) 90 \% \\
(\mathrm{HOMO}-1 \rightarrow \mathrm{LUMO}+1) 8 \%\end{array}$ \\
\hline HL1-Pt & 5 & 332.9 & 0.176 & 3.724 & $\begin{array}{l}\mathrm{P} \rightarrow \mathrm{H}(\mathrm{HOMO} \rightarrow \mathrm{LUMO}) 76 \% \\
(\mathrm{HOMO}-1 \rightarrow \mathrm{LUMO}+1) 22 \%\end{array}$ \\
\hline HL1-Zn & 4 & 271.2 & 0.594 & 4.572 & $\begin{array}{l}\mathrm{P} \rightarrow \mathrm{H}(\mathrm{HOMO} \rightarrow \mathrm{LUMO}) 84 \% \\
(\mathrm{HOMO}-1 \rightarrow \mathrm{LUMO}+1) 11 \%\end{array}$ \\
\hline HL2 & 4 & 297.6 & 1.064 & 4.166 & $\mathrm{P} \rightarrow \mathrm{H}(\mathrm{HOMO} \rightarrow \mathrm{LUMO}) 93 \%$ \\
\hline HL2-Pd & 6 & 398.4 & 0.061 & 3.112 & $\begin{array}{l}\mathrm{P} \rightarrow \mathrm{H}(\mathrm{HOMO} \rightarrow \mathrm{LUMO}) 78 \% \\
(\mathrm{HOMO}-1 \rightarrow \mathrm{LUMO}+1) 21 \%\end{array}$ \\
\hline HL2-Pt & 5 & 418.2 & 0.071 & 2.965 & $\mathrm{P} \rightarrow \mathrm{H}(\mathrm{HOMO} \rightarrow \mathrm{LUMO}) 94 \%$ \\
\hline HL2-Zn & 6 & 281.8 & 0.415 & 4.400 & $\begin{array}{l}\mathrm{P} \rightarrow \mathrm{H}(\mathrm{HOMO} \rightarrow \mathrm{LUMO}) 80 \% \\
(\mathrm{HOMO}-1 \rightarrow \mathrm{LUMO}+1) 17 \%\end{array}$ \\
\hline
\end{tabular}


The maximum wavelength bands presented in Table 8 for all the compounds have been found to originate from the almost degenerated HOMO/HOMO - 1 couple to the LUMO and LUMO + 1 respectively. However, in all the compounds studied, the HOMO to LUMO transition is the main contribution to the absorption spectra. The HL1 ligand is seen to possess a maximum absorption band that appears around $251.4 \mathrm{~nm}$. It is worthy of note that the maximum absorption peak of HL1 shows a bathochromic shift with the introduction of the electron withdrawing nitro group in HL2. Also, upon complexation of both ligands, a bathochromic shift is observed with the exception of the HL2-Zn complex that witnesses a hypsochromic shift. On the whole, it is observed that apart from the HL2-Pt complex, all the studied compounds show good transparency tradeoffs in the visible region for NLO efficiency.

In a bid to decipher the nature and origin of electronic excitation, the isosurfaces of the hole, particle and particle-hole overlap as shown in Table 9 have been generated via the Multiwfn 3.6(dev) software [49] and visualized using the Chemcraft 1.8 visualization software [50]. It is observed that $\pi \rightarrow \pi^{*}$ electronic transitions are predominant in the chalcone moiety of HL1 and HL2 ligands while in the studied complexes, we have some low-lying $d \rightarrow \pi^{*}$ and $\pi \rightarrow d$ or $d \rightarrow d$ transitions, although bands with larger intensity are in general due to charge-transfer transitions. Also, the particle-hole overlap in the HL2 ligand is found to be more extensive than the overlap in the HL1 ligand. Hence, nitration enhances charge transfer (CT) in HL1 which explains the impressive increment of its $\beta$ and $\gamma$ values observed. In the Pt and Pd complexes it is observed that particle-hole overlaps are mainly localized around the metal-based d-orbitals, the chloride ligand and the phenolic ring of HL2 complexes.

Table 9. The dominant NTO "particle-hole" pairs for the dominant electronic transitions of each investigated molecule and the "particle-hole" overlap in gas-phase.

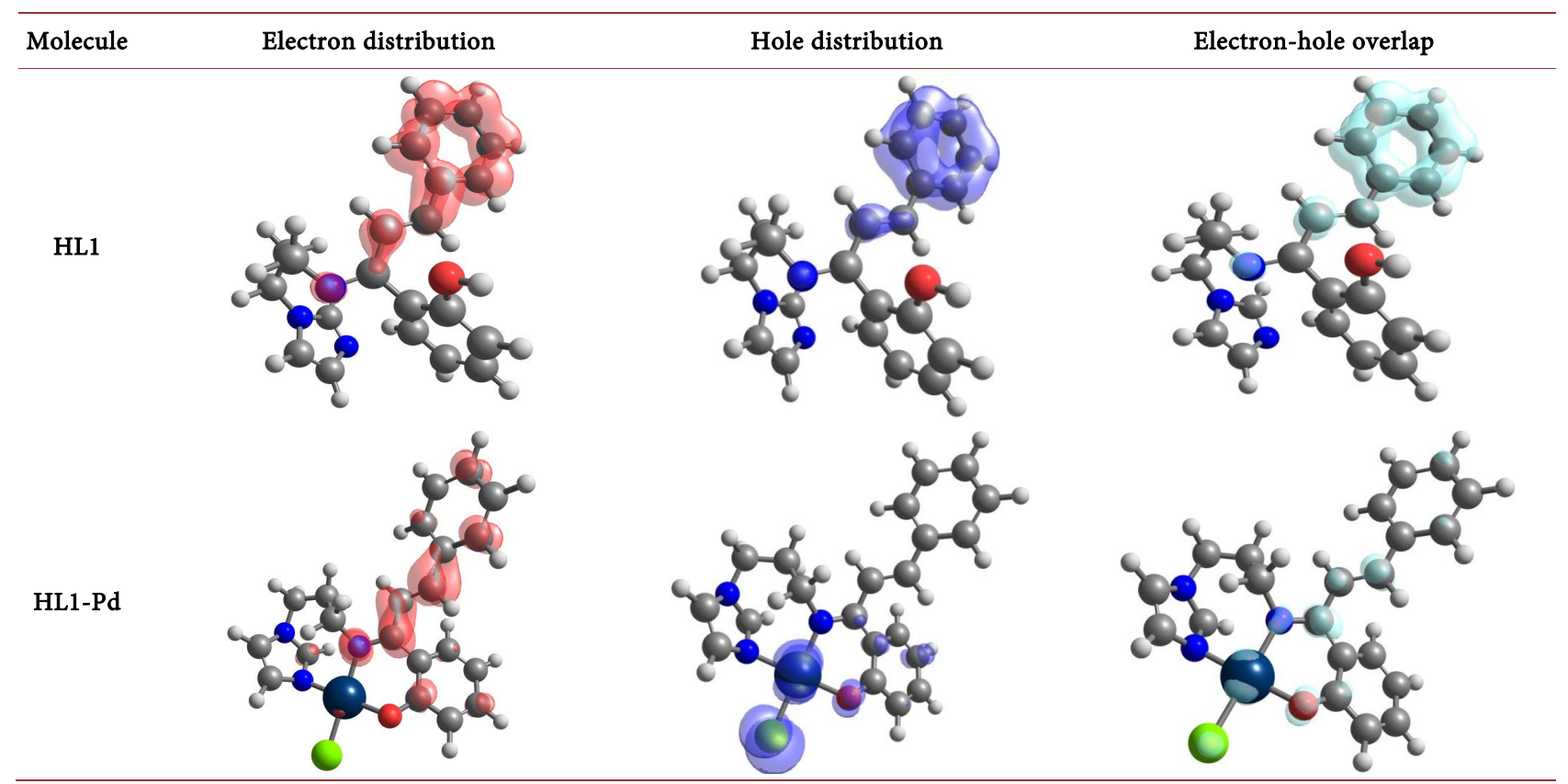


HL1-Pt

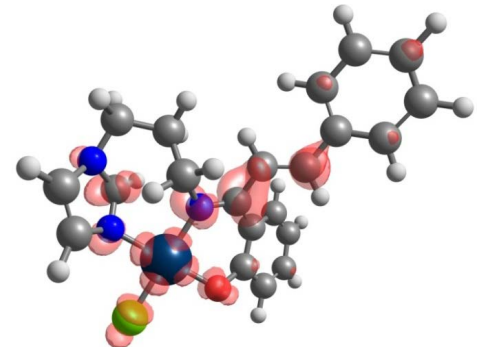

LL1-Zn

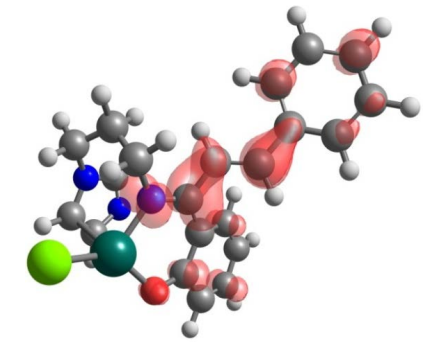

HL2

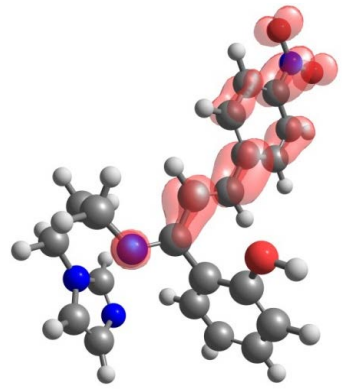

HL2-Pt
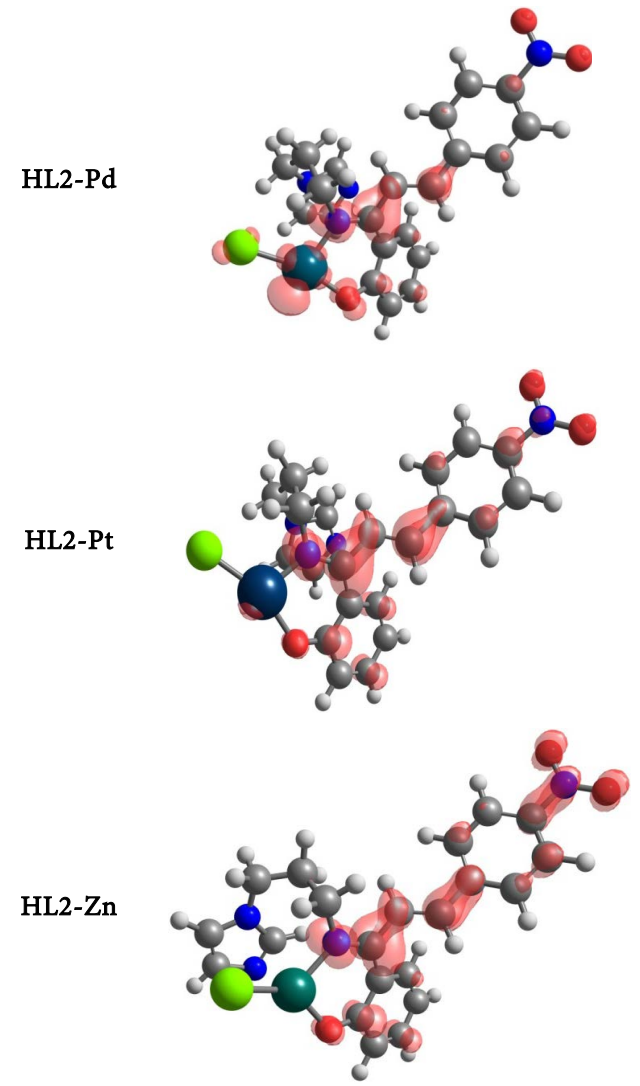
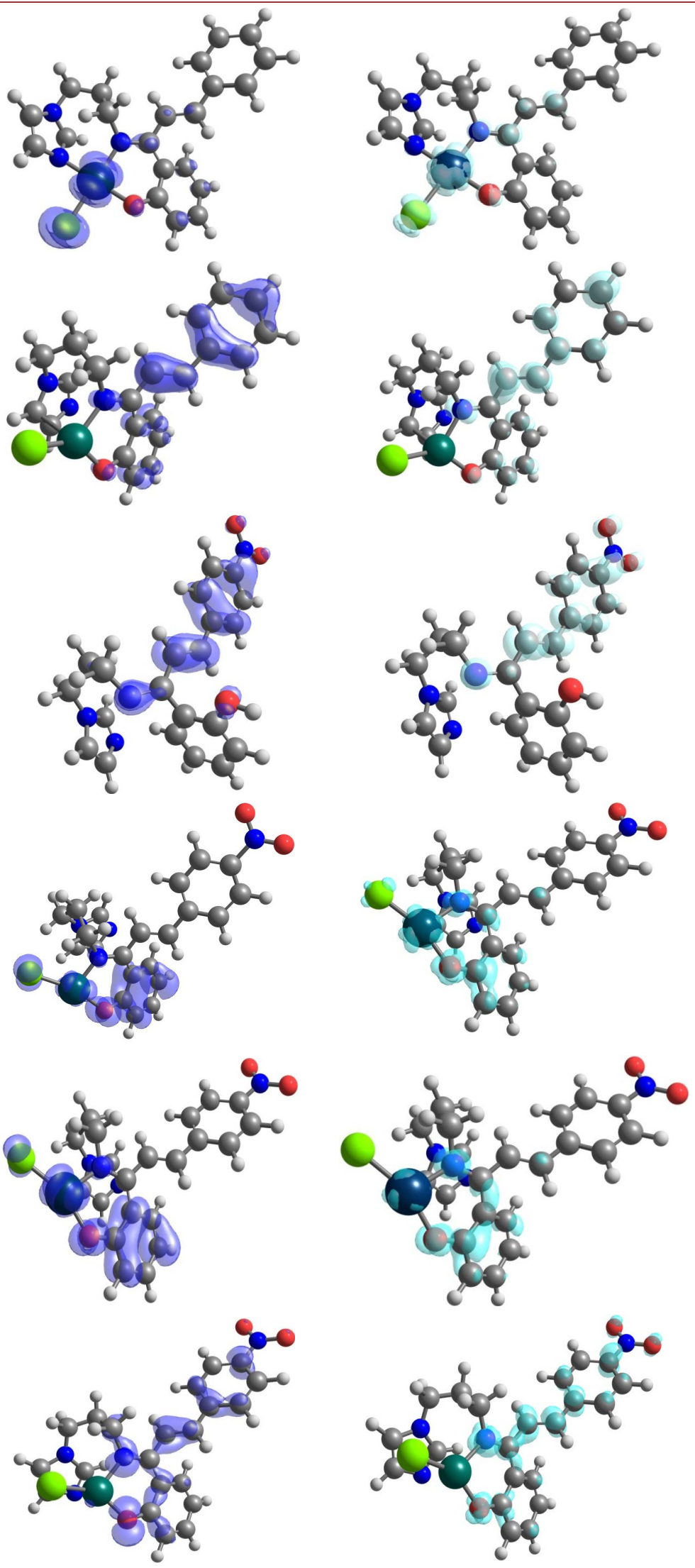
This observation is evident of metal-ligand charge transfer (MLCT) and ligandmetal charge transfer (LMCT) dominated electronic transitions within the $\mathrm{Pt}(\mathrm{II})$ and $\mathrm{Pd}$ (II) metal chlorides complexes studied (except the $\mathrm{Zn}$ (II) chloride complex), along with inherent ligand-ligand charge transfer (LLCT) transitions. The introduction of MLCT and LMCT electronic transitions often, but not always, boosts the first molecular hyperpolarizabilities of organic push-pull systems, as observed in the present study. This observation nicely corroborates the relatively lower hyperpolarizabilities of the $\mathrm{Zn}(\mathrm{II})$ complexes compared to their Pt(II) and Pd(II) counterparts as charge transfer in the $\mathrm{Zn}(\mathrm{II})$ complexes is observed to be essentially LLCT.

\section{Conclusion}

Chalcone based ligands have widely been investigated for optoelectronic and photonic applications. Nevertheless, chalcone-imidazole based metal-organic compounds have been paid less attention. In the present work therefore, theoretical investigations via the density functional theory (DFT) method have been undertaken in a bid to predict the combined effects of the nitro group substituent and complexation on the electron/hole-transport properties and the nonlinear optical (NLO) activity of two imidazole-based chalcone ligands: 2-[1-(3(1H-imidazol-1-yl)propylimino)-3-(phenylallyl)]phenol and 2-[1-(3-(1H-imidazol-1-yl)propylimino)-3-4-nitro-phenylallyl]phenol. From our results, the $\mathrm{Pd}(\mathrm{II})$ and Pt(II) complexes have been found to exhibit excellent hole-transport properties, owing to their very small reorganization energies, suggestive of the fact that these complexes could serve as promising charge transport materials suitable for the manufacture of organic light emitting diodes (OLEDs). The light extraction efficiency of the HL1-Pt complex was deduced to be particularly impressive, thus suitable for the manufacture of hole transport layer in violet light emitting diodes (LEDs). Moreover, redox potentials and chemical stability studies have been used to attest for the greater stability in moisture of HL2 complexes towards quenching compared to their HL1 counterparts. The first and second hyperpolarizabilities of the HL1-Pd and HL1-Zn complexes have been found to be inferior to that of paranitroaniline. That notwithstanding, the HL1-Pt, the HL2 ligand and all the HL2 complexes have been found to show high prospects to be used as NLO materials given that their $\beta$ and $\gamma$ values are greater than that of the prototype paranitroaniline. Additionally, it has been observed that metal chelation enhances the NLO response of the chalcone-based imidazole ligands investigated in this study. Overall, the studied compounds have demonstrated interesting properties for the manufacture of optoelectronic and photonic devices capable of second and third-order NLO response with good transparency tradeoffs. Base on the forgoing, the present study may serve as a guide for experimental works in the development of highly stable chalcone-imidazole complexes with meaningful nonlinear optical and charge transport properties for practical use.

\section{Acknowledgements}

The authors gratefully acknowledge the material support from the Research 
Unit of Noxious Chemistry and Environmental Engineering of the University of Dschang, Cameroon.

\section{Conflicts of Interest}

The authors declare no conflicts of interest regarding the publication of this paper.

\section{References}

[1] Shkir, M., Irfan, A., AlFaify, S., Patil, P.S. and Al-Sehemi A.G. (2019) Linear, Second and Third Order Nonlinear Optical Properties of Novel noncentrosymmetric Donor-Acceptor Configure Chalcone Derivatives: A Dual Approach Study. Optik International Journal for Light and Electron Optics, 199, Article ID: 163354. https://doi.org/10.1016/j.ijleo.2019.163354

[2] Kumari, R., Varghese, A., George, L. and Sudhakar, Y.N. (2017) Effect of Solvent Polarity on the Photophysical Properties of Chalcone Derivatives. RSC Advances, 7, 24204-24214. https://doi.org/10.1039/C7RA01705G

[3] Kalanithi, M., Rajarajan, M., Tharmaraj, P. and Sheela, C.D. (2012) Spectral, Biological Screening of Metal Chelates of Chalcone Based Schiff Bases of N-(3-aminopropyl)imidazole. Spectrochimica Acta A, 87, 155-162. https://doi.org/10.1016/j.saa.2011.11.031

[4] Custodio, J.M.F., D’Oliveira, G.D.C., Gotardo, F., Cocca, L.H.Z., De Boni, L., Perez, C.N., Maia, L.J.Q., Valverde, C., Osorio, F.A.P. and Napolitano, H.B. (2019) Chalcone as Potential Nonlinear Optical Material: A Combined Theoretical, Structural, and Spectroscopic Study. Journal of Physical Chemistry C, 123, 5931-5941. https://doi.org/10.1021/acs.jpcc.9b01063

[5] Naik, V.S., Patil, P.S., Gummagol, N.B., Wong, Q.A., Quah, C.K. and Jayanna, H.S. (2020) Crystal Structure, Linear and Nonlinear Optical Properties of Three Thiophenyl Chalcone Derivatives: A Combined Experimental and Computational Study. Optical Materials, 110, Article ID: 110462. https://doi.org/10.1016/j.optmat.2020.110462

[6] Prabhu, A.N., Jayarama, A., Subrahmanya, B.K., Manjunatha, K.B., Umesh, G. and Upadhyaya V. (2013) Physicochemical Studies on Thienyl Chalcone Derivative for Nonlinear Optical Application. Indian Journal of Materials Science, 2013, Article ID: 151230. https://doi.org/10.1155/2013/151230

[7] Leung, M.K., Chang, C.C., Wu, M.H., Chuang, K.H., Lee, J.H., Shieh, S.J., Lin, S.C. and Chiu, C.F. (2006) 6-N,N-diphenylaminobenzofuran-Derived Pyran Containing Fluorescent Dyes: A New Class of High Brightness Red-Light-Emitting Dopants for OLED. Organic Letters, 8, 2623-2626. https://doi.org/10.1021/ol060803c

[8] Wei, M.-K., Lin, C.-W., Yang, C.-C., Kiang, Y.-W., Lee, J.-H. and Lin H.-Y. (2010) Emission Characteristics of Organic Light-Emitting Diodes and Organic Thin-Films with Planar and Corrugated Structures. International Journal of Molecular Sciences, 11, 1527-1545. https://doi.org/10.3390/ijms11041527

[9] Zyss, J. (1985) Nonlinear Organic Materials for Integrated Optics-A Review. Journal of Molecular Electronics, 1, 25-45.

[10] Karthi, S. and Girija, E.K. (2014) Synthesis, Growth, Structure Determination and Optical Properties of Chalcone Derivative Single Crystal. AIP Conference Proceedings, 1591, 1188-1190. https://doi.org/10.1063/1.4872898

[11] Jin, R., Zhang, X., Xiao, W. and Luo, D. (2017) Theoretical Investigations of the Pho- 
tophysical Properties of Star-Shaped $\pi$-Conjugated Molecules with Triarylboron Unit for Organic Light-Emitting Diodes Applications. International Journal of Molecular Sciences, 18, 2178. https://doi.org/10.3390/ijms18102178

[12] Islam, N. and Lone, I.H. (2017) Computational Studies on Optoelectronic and Nonlinear Properties of Octaphyrin Derivatives. Frontiers in Chemistry, 5, 11. https://doi.org/10.3389/fchem.2017.00011

[13] Tay, M.G., Tiong, M.H., Chia, Y.Y., Kuan, S.H.C. and Liu, Z.-Q. (2016) A Way to Improve Luminescent Efficiency of Bis-Chalcone Derivatives. Journal of Chemistry, 2016, Article ID: 3608137. https://doi.org/10.1155/2016/3608137

[14] Islam, N. and Pandith, A.H. (2014) Analysis of Vibrational Spectra (FT-IR and VCD) and Nonlinear Optical Properties of $[\mathrm{Ru}(\mathrm{L}) 3] 2+$ complexes. Journal of Coordination Chemistry, 67, 3288-3310. https://doi.org/10.1080/00958972.2014.961921

[15] Fan, D., Yi, Y., Li, Z., Liu, W., Peng, Q. and Shuai, Z. (2015) Solvent Effects on the Optical Spectra and Excited-State Decay of Triphenylamine-Thiadiazole with Hybridized Local Excitation and Intramolecular Charge Transfer. Journal of Physical Chemistry A, 119, 5233-5240. https://doi.org/10.1021/jp5099409

[16] Nkungli, N.K. and Ghogomu, J.N. (2016) Concomitant Effects of Transition Metal Chelation and Solvent Polarity on the First Molecular Hyperpolarizability of 4-Methoxyacetophenone Thiosemicarbazone: A DFT Study. Journal of Theoretical Chemistry, 2016, Article ID: 7909576. https://doi.org/10.1155/2016/7909576

[17] Islam, N. and Chimni, S.S. (2016) DFT Investigation on Nonlinear Optical (NLO) Properties of Novel Borazine Derivatives. Computational and Theoretical Chemistry, 1086, 58-66. https://doi.org/10.1016/j.comptc.2016.04.016

[18] Orio, M., Pantazis, D.A. and Neese, F. (2009) Density Functional Theory. Photosynthesis Research, 102, 443-453. https://doi.org/10.1007/s11120-009-9404-8

[19] Neese, F. (2012) The ORCA Program System. Wiley Interdisciplinary Reviews. Computational Molecular Science, 2, 73-78. https://doi.org/10.1002/wcms.81

[20] Granovsky, A.A. (2016) Firefly Version 8.2.0.

[21] Schmidt, M.W., Baldridge, K.K., Boatz, J.A., Elbert, S.T., Gordon, M.S., Jensen, J.H., Koseki, S., Matsunaga, N., Nguyen, K.A., Su, S., Windus, T.L., Dupuis, M. and Montgomery, J.A. (1993) General Atomic and Molecular Electronic Structure System. Journal of Computational Chemistry, 14, 1347-1363. https://doi.org/10.1002/jcc.540141112

[22] Becke, A.D. (1988) Density-Functional Exchange-Energy Approximation with Correct Asymptotic Behaviour. Physical Reviews, 38, 3098-3100. https://doi.org/10.1103/PhysRevA.38.3098

[23] Perdew, J.P. (1986) Density-Functional Approximation for the Correlation Energy of the Inhomogeneous Electron gas. Physical Reviews B, 34, 8822-8824. https://doi.org/10.1103/PhysRevB.33.8822

[24] Weigend, F. and Ahlrichs, R. (2005) Balanced Basis Sets of Split-Valence, Triple Zeta Valence and Quadruple Zeta Valence Quality for H to Rn: Design and Assessment of Accuracy. Physical Chemistry Chemical Physics, 7, 3297-3305. https://doi.org/10.1039/b508541a

[25] Brandenburg, J.G., Bannwarth, C., Hansen, A. and Grimme, S. (2018) B97-3c: A Revised Low-Cost Variant of the B97-D Density Functional Method. The Journal of Chemical Physics, 148, Article ID: 064104. https://doi.org/10.1063/1.5012601

[26] Neese, F., Wennmohs, F., Hansen, A. and Becker, U. (2009) Efficient, Approximate and Parallel Hartree-Fock and Hybrid DFT Calculations. A "Chain-of-Spheres" Algo- 
rithm for the Hartree-Fock Exchange. Chemical Physics, 356, 98-109. https://doi.org/10.1016/j.chemphys.2008.10.036

[27] Neese, F. (2003) An Improvement of the Resolution of the Identity Approximation for the Calculation of the Coulomb Matrix. Journal of Computational Chemistry, 24, 1740-1747. https://doi.org/10.1002/jcc.10318

[28] Yanai, T., Tew, D.P. and Handy, N.C. (2004) A New Hybrid Exchange-Correlation Functional Using the Coulomb-Attenuating Method (CAM-B3LYP). Chemical Physics Letters, 393, 51-57. https://doi.org/10.1016/j.cplett.2004.06.011

[29] Adamo, C. and Jacquemin, D. (2013) The Calculations of Excited-State Properties with Time-Dependent Density Functional Theory. Chemical Society Reviews, 42, 845 856. https://doi.org/10.1039/C2CS35394F

[30] Perdew, J.P., Ernzerhof, M. and Burke, K. (1996) Rationale for Mixing Exact Exchange with Density Functional Approximations. Journal of Chemical Physics, 105, 9982-9985. https://doi.org/10.1063/1.472933

[31] Guezguez, I., Karakas, A., Iliopoulos, K., Derkowska-Zielinska, B., El-Ghayoury, A., Ranganathan, P., Batail, A., Migalska-Zalas, B., Sahraoui and Karakaya, M. (2013) Theoretical and Experimental Investigations on the Nonlinear Optical Properties of Gold(III) Dithiolene Complexes. Optical Materials, 36, 106-111.

https://doi.org/10.1016/j.optmat.2013.04.037

[32] Grimme, S., Antony, J., Ehrlich, S. and Krieg, H. (2010) A Consistent and Accurate $a b$ Initio Parametrization of Density Functional Dispersion Correction (DFT-D) for the 94 Elements H-Pu. The Journal of Chemical Physics, 132, Article ID: 154104. https://doi.org/10.1063/1.3382344

[33] Grimme, S., Ehrlich, S. and Goerigk, L. (2011) Effect of the Damping Function in Dispersion Corrected Density Functional Theory. Journal of Computational Chemistry, 32, 1456-1465. https://doi.org/10.1002/jcc.21759

[34] Allen, F.H., Kennard, O., Watson, D.G., Brammer, L., Orpen, A.G. and Taylor, R. (1987) Tables of Bond Lengths Determined by X-Ray and Neutron Diffraction. Part 1. Bond Lengths in Organic Compounds. Journal of Chemical Society, Perkin Transactions, 2, S1-S19. https://doi.org/10.1039/p298700000s1

[35] Orpen, A.G., Brammer, L., Allen, F.H., Kennard, O., Watson, D.G. and Taylor, R. (1989) Tables of Bond Lengths Determined by X-Ray and Neutron Diffraction. Part 2. Organometallic Compounds and Coordination Complexes of the d-and f-Block Metals. Journal of Chemical Society, Dalton Transactions, 12, S1-S83. https://doi.org/10.1039/dt98900000s1

[36] Stallinga, P. (2011) Electronic Transport in Organic Materials: Comparison of Band Theory with Percolation/ (Variable Range) Hopping Theory. Advanced Materials, 23, 3356-3362. https://doi.org/10.1002/adma.201101129

[37] Marcus, R.A. (1957) On the Theory of Oxidation-Reduction Reactions Involving Electron Transfer. II. Applications to Data on the Rates of Isotopic Exchange Reactions. The Journal of Chemical Physics, 26, 867-871.

https://doi.org/10.1063/1.1743423

[38] Marcus, R.A. (1957) On the Theory of Oxidation-Reduction Reactions Involving Electron Transfer. III. Applications to Data on the Rates of Organic Redox Reactions. The Journal of Chemical Physics, 26, 872-877. https://doi.org/10.1063/1.1743423

[39] Shuai, Z., Wang, L. and Song, C. (2012) Theory of Charge Transport in Carbon Electronic Materials. Springer Briefs in Molecular Science, 9, 90.

https://doi.org/10.1007/978-3-642-25076-7 
[40] Sun, F. and Jin, R. (2013) DFT and TD-DFT Study on the Optical and Electronic Properties of Derivatives of 1,4-bis(2-substituted-1,3,4-oxadiazole)benzene. Arabian Journal of Chemistry, 73, 1-6.

[41] Domingo, L.R. (2016) Molecular Electron Density Theory: A Modern View of Reactivity in Organic Chemistry. Molecules, 21, 1-15.

https://doi.org/10.3390/molecules21101319

[42] Ervin, K.M., Anusiewicz, I., Skurski, P., Simons, J. and Lineberger, W.C. (2003) The Only Stable State of $\mathrm{O}_{2}$-Is the $\mathrm{X} \Pi_{\mathrm{g}}$ Ground State and It (Still!) Has an Adiabatic Electron Detachment Energy of $0.45 \mathrm{eV}$. The Journal of Physical Chemistry A, 107, 8521-8529. https://doi.org/10.1021/jp0357323

[43] Pandith, A.H. and Islam, N. (2014) Electron Transport and Nonlinear Optical Properties of Substituted Aryldimesityl Boranes: A DFT Study. PLOS ONE, 9, e114125. https://doi.org/10.1371/journal.pone.0114125

[44] Park, S.M., Mazza, S.M., Liang, Z., Abtahi, A., Boehm, A.M., Parkin, S.R., Anthony, J.E. and Graham, K.R. (2018) Processing Dependent Influence of the Hole Transport Layer IonizationEnergy on Methylammonium Lead Iodide Perovskite Photovoltaics. ACS Applied Materials \& Interfaces, 10, 15548-15557.

https://doi.org/10.1021/acsami.7b16894

[45] Ghogomu, J.N. and Nkungli, N.K. (2016) A DFT Study of Some Structural and Spectral Properties of 4-Methoxyacetophenone Thiosemicarbazone and Its Complexes with Some Transition Metal Chlorides: Potent Antimicrobial Agents. Advances in Chemistry, 2016, Article ID: 9683630. https://doi.org/10.1155/2016/9683630

[46] Nie, W. (1993) Optical Nonlinearity: Phenomena, Applications, and Materials. Advanced Materials, 5, 520-545. https://doi.org/10.1002/adma.19930050704

[47] Bosshard, C., Bösch M., Liakatas, I., Jäger, M. and Günter, P. (2000) Second-Order Nonlinear Optical Organic Materials: Recent Developments. In: Günter, P., Ed., Nonlinear Optical Effects and Materials, Springer Series in Optical Sciences, Vol. 72, Springer, Berlin, 163-299. https://doi.org/10.1007/978-3-540-49713-4 3

[48] Oudar, J.L. and Chemla, D.S. (1977) Hyperpolarizabilities of the Nitroanilines and Their Relations to the Excited State Dipole Moment. The Journal of Chemical Physics, 66, 2664-2668. https://doi.org/10.1063/1.434213

[49] Lu, T. and Chen, F. (2011) Multiwfn: A Multifunctional Wavefunction Analyzer. Journal of Computational Chemistry, 33, 580-592. https://doi.org/10.1002/jcc.22885

[50] Zhurko, G.A. Chemcraft-Graphical Program for Visualization of Quantum Chemistry Computations. https://chemcraftprog.com 